\title{
Performance Modelling of a 1 kJ DPSSL System
}

\author{
K. Ertel, S. Banerjee, C. Hernandez-Gomez, P. D. Mason, P. J. Phillips, J. L. Collier \\ STFC Rutherford Appleton Laboratory, Central Laser Facility, Chilton, Didcot, OX11 OQX, United Kingdom \\ klaus.ertel@stfc.ac.uk
}

\begin{abstract}
We present modelling results for a $1 \mathrm{~kJ}$ diode-pumped laser system, based on cryogenic gas-cooled multi-slab Yb:YAG amplifiers.

OCIS codes: (140.3280) Laser amplifiers; (140.3480) Lasers, diode-pumped, (140.3615) Lasers, ytterbium
\end{abstract}

\section{Introduction}

More and more projects and applications require the development of kJ-class laser systems operating at multi-Hz repetition rate and high wall-plug efficiency, which is only possible using diode pumped solid state laser (DPSSL) technology. These lasers will either be used directly for generating plasmas, e.g. in the context of inertial fusion energy (IFE) production, or for pumping short-pulse amplifiers such as OPCPA or Ti:Sapphire systems. The PW to multi-PW peak power pulses generated from these systems will be used for fundamental studies and to develop new applications such as laser-driven particle accelerators. Two large scale European research projects are underway to develop and exploit such high-energy, high-repetition rate laser sources. They are ELI [1], dedicated to ultra-short pulse laser research and applications, and HiPER [2], focussed on IFE research.

In [3] we have presented a concept for a kJ-class DPSSL amplifier, based on cryogenic gas cooled multi-slab $\mathrm{Yb}: Y A G$ technology. Fig. 1 shows an illustration of the amplifier geometry and Table 1 lists design and calculated performance parameters for a baseline configuration. We have explored ways to maximise the amount of extractable energy while minimising ASE losses and keeping pump and extraction fluence at moderate levels, compatible with today's diode technology and with the requirements for reliable long-term operation, e.g. in an IFE power plant. In this paper we will discuss how a laser beamline could be constructed using this amplifier technology and what the expected performance will be, based on numerical modelling.

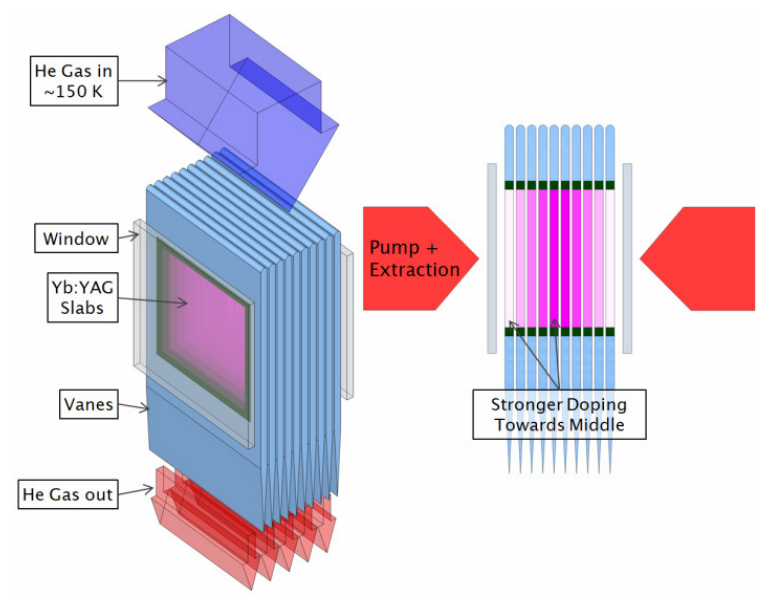

Fig. 1. Illustration of amplifier concept: isometric (left) and side

Table 1. Design and modelled performance parameters of cryo-cooled multi-slab amplifier.

\begin{tabular}{|c|c|}
\hline Target output fluence & $5 \mathrm{~J} / \mathrm{cm}^{2}$ \\
\hline Total pump intensity & $10 \mathrm{~kW} / \mathrm{cm}^{2}$ \\
\hline Pump duration & $1 \mathrm{~ms}$ \\
\hline Pump wavelength & $939 \mathrm{~nm}$ \\
\hline Laser wavelength & $1030 \mathrm{~nm}$ \\
\hline Temperature & $175 \mathrm{~K}$ \\
\hline Storage efficiency & $50 \%\left(5 \mathrm{~J} / \mathrm{cm}^{2}\right.$ stored $)$ \\
\hline Small signal gain & 3.8 \\
\hline Beam size & $14 \times 14 \mathrm{~cm}^{2}$ \\
\hline No. of slabs & 10 \\
\hline Slab thickness & $10 \mathrm{~mm}$ \\
\hline
\end{tabular}

\section{Beamline concept}

A full beamline will start off with a front-end oscillator and preamplifier stages, which need to provide laser pulses with appropriate spatial and temporal profiles at about the joule level. These components shall not be discussed here as the technology is not expected to differ significantly from existing high energy laser systems such as LMJ [4] and NIF [5]. The back-end part of the beamline will consist of one or more power amplifier modules and a suitable optical architecture for energy extraction and beam transport. Our chosen concept is similar to that employed on LMJ and also on the Mercury laser [6]. It consists of two identical amplifier heads, connected in series, which are passed four times by the extraction pulse (the laser pulse to be amplified). This is achieved through angular multiplexing in conjunction with a spatial filter at the end of the beamline. The beam is injected near the focus of the spatial filter and also picked off and re-injected after the second pass. A schematic block diagram of the beamline concept is shown in Fig. 2. The optical losses assumed for the baseline model scenario are also indicated in Fig. 2. A loss of $1 \%$ per amplifier slab was assumed and an additional $10 \%$ at the end of each pass to account for losses on 


\section{HThE3.pdf}

other components such as windows, spatial filters, mirrors, polarisers and Pockels cells. These assumptions are naturally quite crude, but they provide a starting point for numeric modelling studies in order to explore parameters such as optical-to-optical efficiency, B-Integral and output pulse shape.

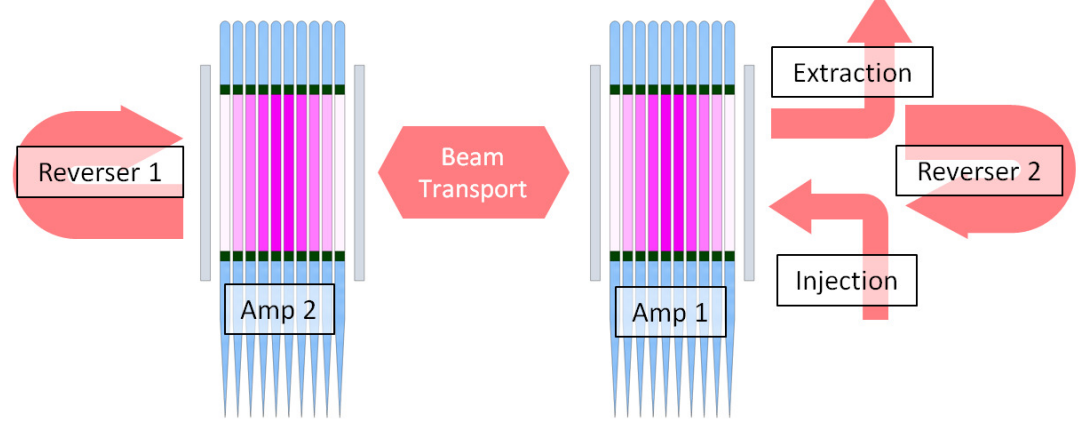

\begin{tabular}{|c|c|c|c|c|c|c|c|c|}
\hline Injection & Amp $1+2$ & Reverser 1 & Amp1 + 2 & Reverser 2 & Amp $1+2$ & Reverser 1 & Amp1 + 2 & Extraction \\
\hline Losses: & $\begin{array}{c}17.4 \% \\
\text { (distributed) }\end{array}$ & $10 \%$ & $\begin{array}{c}17.4 \% \\
\text { (distributed) }\end{array}$ & $10 \%$ & $\begin{array}{c}17.4 \% \\
\text { (distributed) }\end{array}$ & $10 \%$ & $\begin{array}{c}17.4 \% \\
\text { (distributed) }\end{array}$ & $10 \%$ \\
\hline
\end{tabular}

Fig. 2. Beamline concept and assumed optical losses.

\section{Modelling results}

We first modelled the generation of a 1-kJ pulse with a duration of $4 \mathrm{~ns}$ and a top-hat shape in time, an approximate representation of the pulse shape required for IFE and OPCPA pumping applications. The results of the calculation are shown in Fig. 3. We can see that the target output pulse energy is reached with an input energy of only $320 \mathrm{~mJ}$. Gain saturation is compensated by an input pulse shape that is exponentially rising toward the trailing edge. Because power at the trailing edge is highest up to the very end, this is where the highest B-Integral value is accumulated. B-Integral averaged over the entire pulse duration amounts to 0.93 . Only the YAG slabs with a total thickness of $20 \mathrm{~cm}$ and a nonlinear index of $6.9 * 10^{16} \mathrm{~cm}^{2} / \mathrm{W}$ were taken into account for the B-Integral calculation.
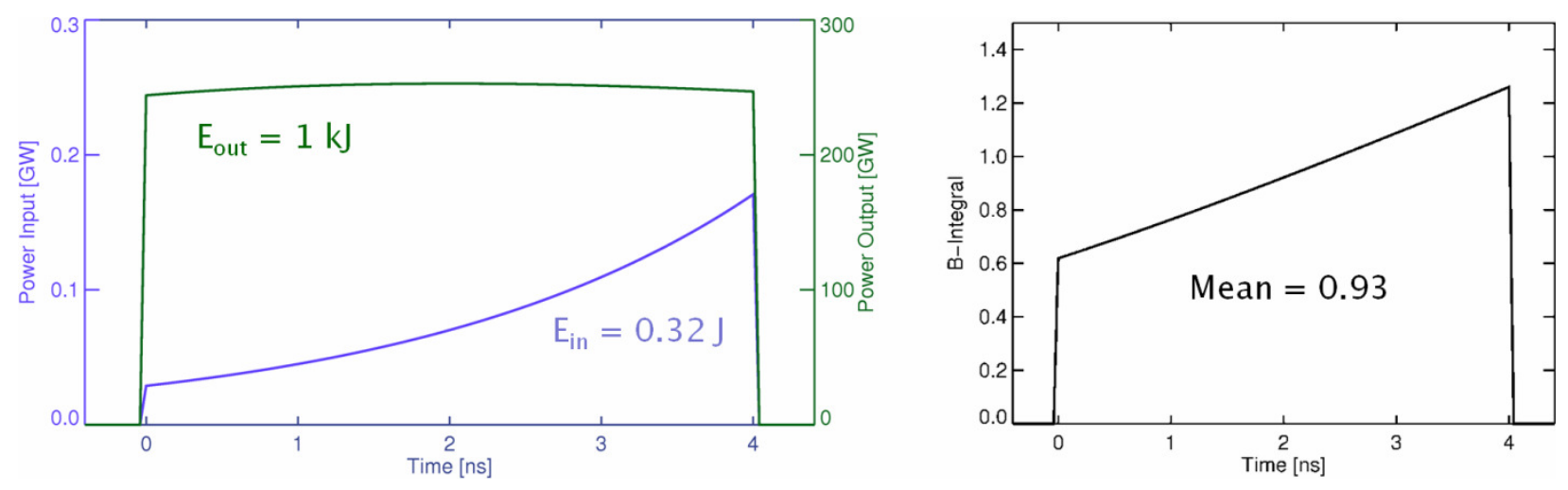

Fig. 3. Left panel: energies and pulse shapes of input (blue) and output (green) pulses. Right panel: B-Integral of output pulse.

The total pump energy in the scenario modelled above is $4 \mathrm{~kJ}$, resulting in an optical-to-optical efficiency of $25 \%$, without taking into account losses in the pump delivery optics, ASE losses, and non-perfect overlap between pump and extraction beams. Considering the electrical-to-optical efficiency of the pump diodes and of the downstream components, harmonic generators in particular, it becomes doubtful if a $10 \%$ overall wall-plug efficiency can be achieved, a figure that is often quoted as required for economically viable IFE generation [7]. Therefore efficiency gains need to be sought. One option is to reduce the diode pump pulse duration, leading to lower fluorescence losses, but resulting in a higher number of required diodes and hence in higher cost. Other ways to gain efficiency are the reduction of optical losses in the beamline and to extract more of the stored energy by operating at a higher accumulated fluence. Table 2 lists parameters for the baseline scenario and a scenario optimised for efficiency. As a result of the lower losses and the higher input energy, the extraction efficiency can be improved to an extent where the same output energy is reached by simply reducing the pump pulse duration, with only a marginal increase in the number of pump diodes required. Because of the lower gain, the amplifiers can also be made thinner without 


\section{HThE3.pdf}

increasing ASE losses, which in turn limits the increase in B-Integral to a moderate value. If higher output fluence and B-Integral values were permissible, the efficiency could be increased by a further few percentage points. Increasing the pump energy to $3 \mathrm{~kJ}$ would yield an output of $1.3 \mathrm{~kJ}$, efficiency of $43 \%$ and B-Integral of 2.3 .

Table 2. Parameters and results for different beamline scenarios

\begin{tabular}{|l|l|l|l|l|l|l|l|l|}
\hline Scenario & $\begin{array}{l}\text { Pump } \\
\text { duration }\end{array}$ & $\begin{array}{l}\text { Loss per } \\
\text { slab }\end{array}$ & $\begin{array}{l}\text { Loss after } \\
\text { each pass }\end{array}$ & $\begin{array}{l}\text { Input } \\
\text { energy }\end{array}$ & $\begin{array}{l}\text { Output } \\
\text { energy }\end{array}$ & $\begin{array}{l}\text { Pump } \\
\text { energy }\end{array}$ & Efficiency & B-Integral \\
\hline Baseline & $1 \mathrm{~ms}$ & $1 \%$ & $10 \%$ & $0.32 \mathrm{~J}$ & $1 \mathrm{~kJ}$ & $4.0 \mathrm{~kJ}$ & $25 \%$ & 0.93 \\
\hline Optimised & $0.6 \mathrm{~ms}$ & $0.5 \%$ & $5 \%$ & $10 \mathrm{~J}$ & $1 \mathrm{~kJ}$ & $2.5 \mathrm{~kJ}$ & $40 \%$ & 1.2 \\
\hline
\end{tabular}

Minimising the optical losses in the back-end section is therefore of high importance for obtaining high extraction efficiency. Another parameter influencing system efficiency is the operating temperature of the amplifier. So far we have concentrated on operation at $175 \mathrm{~K}$ and at $300 \mathrm{~K}$ because of the availability of spectrally resolved pump absorption cross section data. Replacing spectrally resolved data by effective cross sections that yield the same efficiencies and extrapolating these allows modelling performance at other temperatures. This time, the input energy was set to $10 \mathrm{~J}$ and the pump energy was adjusted such that an output of $1 \mathrm{~kJ}$ was obtained. The calculations were again carried out for two scenarios, with losses and pump duration as listed in Table 2. The results are shown in Fig. 4. They confirm that operating at low temperature gives a significant increase in optical-to-optical efficiency, due to a combination of reduced reabsorption loss and increased gain cross section. The beneficial effect of reducing optical losses is enhanced at low temperatures as gain saturation occurs at lower fluence levels.

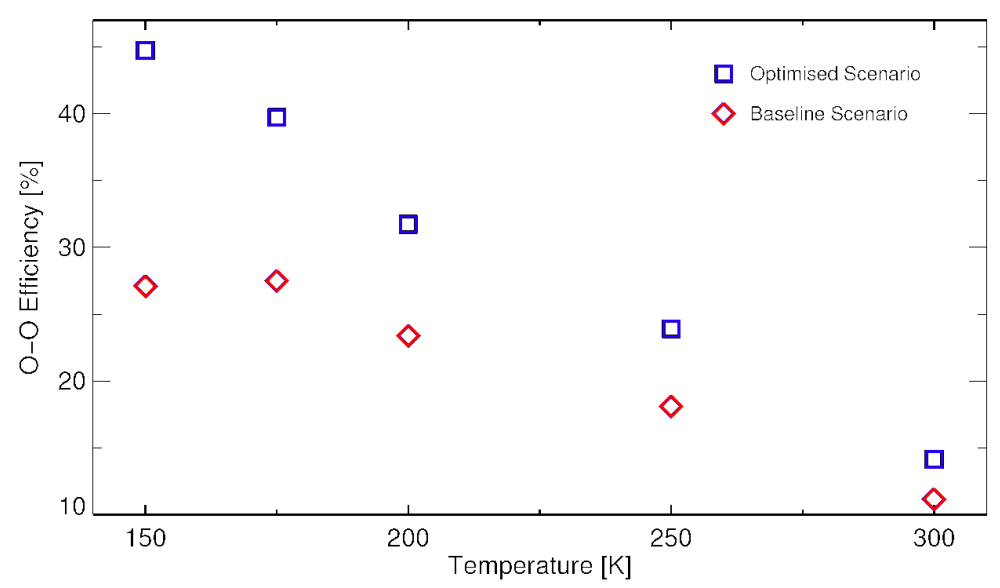

In conclusion, we have demonstrated that very high optical-to-optical efficiency levels should be achievable in a cryogenic Yb:YAG amplifier and that the proposed multi-slab architecture can be scaled at least to the $1 \mathrm{~kJ}$ level. The ultimate limit to scaling is growing B-Integral as the gain medium needs to become thicker for larger apertures while reducing the doping concentration in order to keep ASE losses at an acceptable level.

\section{References}

[1] The Extreme Light Infrastructure European Project, http://www.extreme-light-infrastructure.eu/eli-home.php.

[2] HiPER Project, http://www.hiper-laser.org.

[3] K. Ertel, C. Hernandez-Gomez, P. D. Mason, I. O. Musgrave, I. N. Ross, and J. L. Collier, "Concept for Cryogenic kJ-Class Yb:YAG Amplifier," in Advanced Solid-State Photonics, OSA Technical Digest Series (CD) (Optical Society of America, 2010), paper AWB20.

[4] M. Andre, "The French Megajoule Laser Project (LMJ)," Fusion Engineering and Design 44, 43-49 (1999).

[5] G. Miller, E. Moses, and C. Wuest, "The National Ignition Facility," Optical Engineering 43, 2841-2853 (2004).

[6] A. Bayramian et al, "The mercury project: A high average power, gas-cooled laser for inertial fusion energy development," Fusion Sci Technol 52, 383-387 (2007).

[7] J. A. Caird et al, “Nd:Glass Laser Design for Laser ICF Fission Energy”, LLNL-CONF-408600, Proc. $18^{\text {th }}$ TOFE, Sept $18^{\text {th }}-$ Oct $2^{\text {nd }}$, San Francisco, CA (2008). 